\title{
Room-temperature ferromagneticlike behavior in Mn-implanted and postannealed InAs layers deposited by molecular beam epitaxy
}

\author{
R. González-Arrabal, ${ }^{1}$ Y. González, ${ }^{1}$ L. González, ${ }^{1}$ M. García-Hernández, ${ }^{2}$ F. Munnik, ${ }^{3}$ \\ and M. S. Martín-González ${ }^{1, a)}$ \\ ${ }_{1}^{1}$ Instituto de Microelectrónica de Madrid CSIC C/Isaac Newton, 8. Tres Cantos, E-28760 Madrid, Spain \\ ${ }^{2}$ Instituto de Ciencia de Materiales de Madrid (CSIC), Cantoblanco, E-28049 Madrid, Spain \\ ${ }^{3}$ Institute of Ion Beam Physics and Materials Research, Forschungszentrum Dresden-Rossendorf, P.O. Box \\ 510119, D-01314 Dresden, Germany
}

(Received 25 September 2008; accepted 22 January 2009; published online 6 April 2009)

\begin{abstract}
We report on the magnetic and structural properties of Ar- and Mn-implanted InAs epitaxial films grown on GaAs (100) by molecular beam epitaxy and the effect of rapid thermal annealing (RTA) for $30 \mathrm{~s}$ at $750{ }^{\circ} \mathrm{C}$. Channeling particle induced x-ray emission (PIXE) experiments reveal that after $\mathrm{Mn}$ implantation almost all $\mathrm{Mn}$ atoms are substitutional in the In site of the InAs lattice, like in a diluted magnetic semiconductor. All of these samples show diamagnetic behavior. However, after RTA treatment the Mn-InAs films exhibit room-temperature magnetism. According to PIXE measurements the Mn atoms are no longer substitutional. When the same set of experiments was performed with $\mathrm{Ar}$ as implantation ion, all of the layers present diamagnetism without exception. This indicates that the appearance of room-temperature ferromagneticlike behavior in the $\mathrm{Mn}-$ InAs-RTA layer is not related to lattice disorder produced during implantation but to a Mn reaction produced after a short thermal treatment. X-ray diffraction patterns and Rutherford backscattering measurements evidence the segregation of an oxygen-deficient $\mathrm{MnO}_{2}$ phase (nominally $\mathrm{MnO}_{1.94}$ ) in the Mn-InAs-RTA epitaxial layers which might be the origin of the room-temperature ferromagneticlike response observed. (C) 2009 American Institute of Physics.
\end{abstract}

[DOI: $10.1063 / 1.3087477$ ]

\section{INTRODUCTION}

Combining semiconducting and ferromagneticlike (FMlike) properties into the same material provides the opportunity of not only controlling conduction in the traditional way (electronics) but also controlling its quantum spin state. Cooperative effects through spin-exchange interaction such as the tuning of the magnetic properties of the semiconductors by changing their carrier density ${ }^{1}$ open the way toward to the emerging technology known as magnetoelectronics or spintronics.

At this point, diluted magnetic semiconductors (DMSs) have recently been a hot topic in the magnetic semiconductor research. They are based on traditional semiconductors but are doped with transition metals such as $\mathrm{Mn}$. One of the drawbacks of DMSs for industrial applications is its commonly low Curie temperature $\left(T_{C}\right)$. However, theoretical predictions ${ }^{2}$ indicate that $T_{C}$ can be raised above $300 \mathrm{~K}$ for those semiconductors that contain a high concentration of magnetic ions or consist of light elements, like in the case of $\mathrm{Mn}$ or Co doped $\mathrm{ZnO}$. Meanwhile, extensive doubts persist on the origin of their magnetism ${ }^{3-6}$ or on the effective interaction between magnetism and semiconductivity. The III-V semiconductors are the set of materials in which spin injection into nonmagnetic semiconductor and FM control by light and electric fields for low Mn concentrations have been demonstrated. Moreover, this kind of materials has also been

\footnotetext{
${ }^{a)}$ Author to whom correspondence should be addressed. Electronic mail: marisol@imm.cmm.csic.es.
}

reported to posses $T_{C}$ above room temperature, i.e., for $\mathrm{In}_{1-x} \mathrm{Mn}_{x} \mathrm{As}$ samples grown by metal-organic vapor phase epitaxy a $T_{C} \sim 330 \mathrm{~K}$ (Refs 7 and 8 ) has been reported. Although, in some other works an experimental $T_{C}<55 \mathrm{~K}$ has been measured for this phase. ${ }^{9}$ Incorporation of a large amount of magnetic atoms in a controlled way during the growth of III-V semiconductor structures by molecular beam epitaxy (MBE) is a difficult task. Usually, the low equilibrium solubility of transition metals (such as $\mathrm{Mn}$ ) into III-V semiconductors originates surface segregation or phase separation above a certain doping threshold. In the case of InAs, magnetic dopants were successfully introduced during growth for the first time in 1989 by Munekata et al. who deposited $\operatorname{In}_{1-x} \mathrm{Mn}_{x}$ As layers by MBE at relatively low temperatures $\left(200-300{ }^{\circ} \mathrm{C}\right)$ and $\mathrm{Mn}$ concentrations $(x \leq 0.026) .{ }^{10}$ To overcome these difficulties, implantation techniques that allow insertion of magnetic impurities into the semiconductors in a controlled way were started to be used. $^{11-13}$

Even when the $\operatorname{In}_{1-x} \mathrm{Mn}_{x}$ As system has been relatively well characterized, some controversies are still found in literature on the origin of its magnetic properties. $\operatorname{In}_{1-x} \mathrm{Mn}_{x} \mathrm{As}$ layers were found to be FM or paramagnetic depending on the local environment surrounding the $\mathrm{Mn}$ atoms, which in turn depends on the growth procedure. ${ }^{10,14,15}$ For films grown at relatively low temperature $\left(\sim 200{ }^{\circ} \mathrm{C}\right) \mathrm{Mn}$ has been observed to incorporate into the InAs structure occupying the In sit, forming a homogeneous InMnAs alloy which presents a paramagnetic behavior, while for films deposited at tem- 

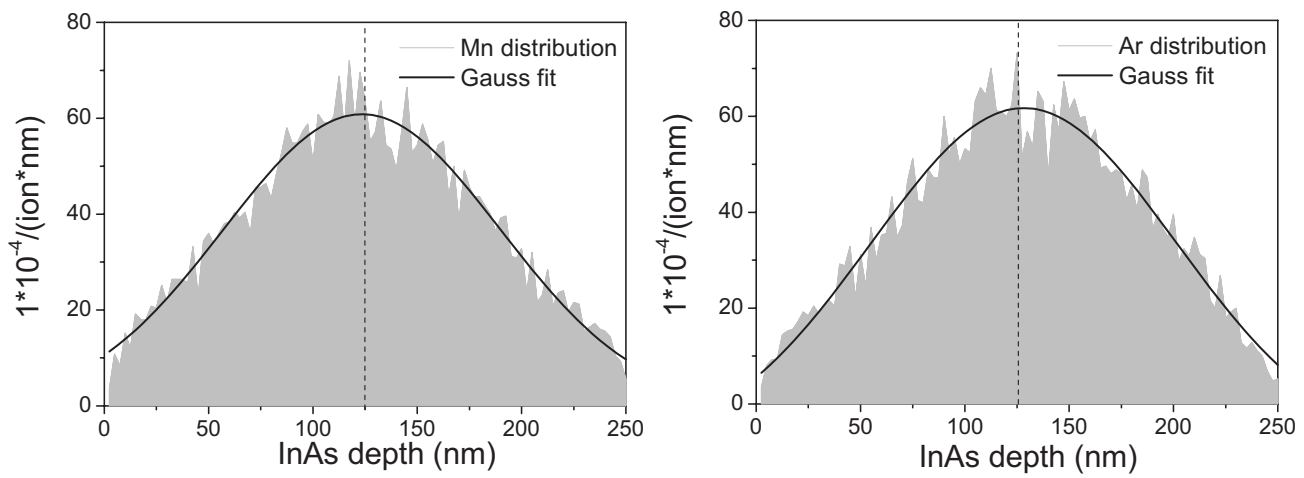

FIG. 1. Mn (Left) and Ar (right) depth distributions within the InAs layer after implantation as simulated by the TRIM Monte Carlo code (Ref. 17)

peratures higher than $300{ }^{\circ} \mathrm{C}$, Mn might form MnAs clusters which are supposed to be responsible for the FM behavior of these films. ${ }^{15}$

To elucidate this point we examine the origin of the magnetism in Mn-implanted InAs epitaxial grown by MBE and the effect of rapid thermal annealing (RTA). The structure of the InAs crystal lattice is investigated for the different processes by x-ray diffraction (XRD). The layer composition has been characterized by Rutherford backscattering spectroscopy (RBS). The relation of magnetism with lattice location of $\mathrm{Mn}$ within the crystal structure is investigated by particle induced $\mathrm{x}$-ray emission (PIXE) and scanning quantum interference device (SQUID) measurements. The appearance of secondary phases in the structure after annealing and its relation with the magnetic properties of the samples is investigated and discussed.

\section{EXPERIMENTAL}

InAs layers with a thickness of $250 \mathrm{~nm}$ were grown on commercial GaAs (100) substrates by atomic layer molecular beam epitaxy (ALMBE) (Ref. 16) at a substrate temperature $T_{s}=440{ }^{\circ} \mathrm{C}$, a growth rate $r_{g}=0.5 \mathrm{ML} / \mathrm{s}$ (monolayers per second), and an arsenic $\left(\mathrm{As}_{4}\right)$ beam equivalent pressure $=2$ $\times 10^{6}$ Torr. Previous to the growth of InAs, a $200 \mathrm{~nm}$ thick GaAs buffer layer was grown at $T_{s}=580{ }^{\circ} \mathrm{C}$ and $r_{g}$ $=1 \mathrm{ML} / \mathrm{s}$ by MBE . According to the reflection high energy electron pattern, a two dimensional InAs growth front is achieved after the first $30 \mathrm{~nm}$ were grown. InAs samples were implanted with $\mathrm{Mn}$ ions at fluences of $1 \times 10^{16}$ and 8 $\times 10^{16} \mathrm{~cm}^{-2}$. InAs samples implanted with Ar ions at a fluence of $1 \times 10^{16} \mathrm{~cm}^{-2}$ have also been studied for comparison purposes. In order to avoid implantation-induced sputtering of the InAs layer, the Mn implantation at high dose (8 $\times 10^{16} \mathrm{~cm}^{-2}$ ) was sequentially carried out in four steps, each of them at a fluence of $2 \times 10^{16} \mathrm{~cm}^{-2}$. The implantations were performed at room temperature and out of channeling incidence. The implantation conditions were selected to achieve a similar damage pattern by implanting with $\mathrm{Mn}$ and with Ar. The implantation energies were 190 and $180 \mathrm{KeV}$ for $\mathrm{Mn}$ and for Ar, respectively. Samples were RTA in Ar atmosphere for $30 \mathrm{~s}$ at a temperature of $750{ }^{\circ} \mathrm{C}$. During annealing, the sample surface was covered with an InAs/GaAs sample to prevent arsenic evaporation.
Structural characterization of the samples was carried out by XRD using a Philips X-PERT four cycle diffractometer with a $\mathrm{Cu} K \alpha$ radiation source. The XRD measurements were performed in Bragg-Brentano geometry.

The layer composition has been characterized by RBS. RBS measurements were performed using very small currents of $\sim 2 \mathrm{nA}$ of a $2 \mathrm{MeV} \mathrm{He}^{+}$beam and placing a standard Si surface barrier detector at $170^{\circ}$ with respect to the beam direction.

Proton induced x-ray emission (PIXE) measurements in channeling configuration were carried out to study the lattice location of the Mn atoms within the InAs host matrix. PIXE angular scan curves were measured using a $\mathrm{H}^{+}$beam at an energy of $1.5 \mathrm{MeV}$. The beam current for PIXE measurements was close to $1 \mathrm{nA}$ and the spot size was about 1.0 $\times 1.2 \mathrm{~mm}^{2}$. The total beam fluence for a single analysis scan was around $140 \mathrm{nC}$ to avoid radiation-induced damage during the measurements. Characteristic $\mathrm{x}$ rays were measured by a $\mathrm{Si}(\mathrm{Li})$ detector located at an angle of $135^{\circ}$ with respect to the beam direction. The position of the $\mathrm{Mn}$ atoms in the host matrix has been determined by comparing the shape and position of the Mn angular scan curve with those for In. The lattice disorder and the percentage of host atoms displaced after implantation, as well as the damage recovery after RTA, have been characterized by the minimum yield $\left(\chi_{\min }\right)$ from the measured PIXE angular scan curves. The minimum yield is the ratio of the yield measured in the channeling direction to that measured in random configuration. $\chi_{\min }$ scales with lattice disorder, being a good indicator of it.

Magnetic characterization of the samples was performed by SQUID. The temperature dependence of the magnetization at constant field and the field dependence at constant temperature were measured in the zero field cooled protocol. The field dependence of the magnetization was measured at temperatures of 5 and $300 \mathrm{~K}$.

\section{RESULTS AND DISCUSSION}

The projected ranges of the implanted $\mathrm{Mn}$ and $\mathrm{Ar}$ ions are calculated with the TRIM code ${ }^{17}$ to be approximately the same for Mn and Ar. As shown in Fig. 1 the elemental depth distributions of these elements within the InAs film are almost identical, presenting a Gaussian-like shape with the center of the Gaussian located at the center of the film (125 $\mathrm{nm})$. The maximum numbers of target atom displacements 


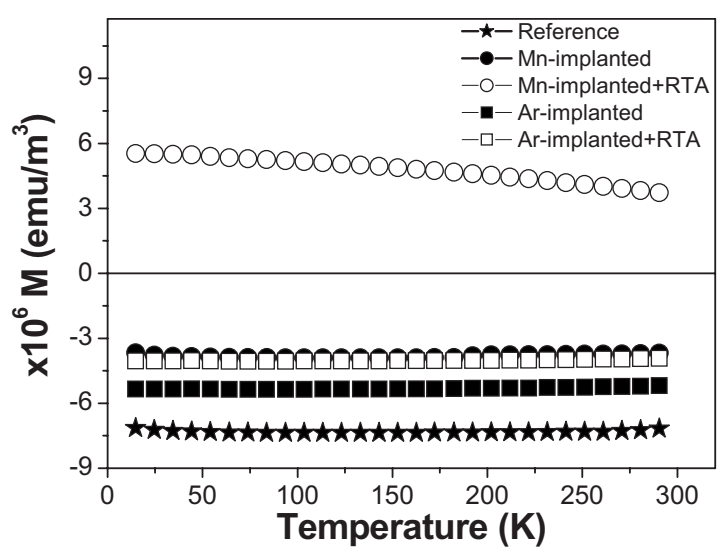

FIG. 2. Temperature dependence of the magnetization at constant field for the reference (black stairs), Mn-implanted (black circles), Mn-RTA (open circles), Ar-implanted (black squares), and Ar-RTA (open squares) samples.

per ion are calculated to be 3.7 and 2.2 for $\mathrm{Mn}$ - and Arimplanted layers, respectively.

The temperature dependence of the magnetization at constant field for the as-grown (reference), Mn-implanted, and Mn-implanted+RTA (from now on Mn-RTA) as well as for Ar-implanted and Ar-RTA samples is shown in Fig. 2. The reference film exhibits a diamagnetic behavior with a magnetic susceptibility of around $-3 \times 10^{-6} \mathrm{emu} / \mathrm{g}$ Oe. Similar behavior is observed for Mn-implanted, Arimplanted, and Ar-RTA samples. Notice that, the diamagnetic response seems to decrease when samples are implanted. We relate the observed behavior to the implantation-induced damage as evidenced in the broadening of the (200) InAs peak (see Fig. 4) and because a similar effect is observed for the Ar-implanted samples. The Mn-RTA sample is the only one presenting positive magnetic susceptibility values for the whole temperature studied range. The fact that at $300 \mathrm{~K}$ the magnetization for the Mn-RTA sample drops to about $68 \%$ of its low temperature value would imply that the Curie temperature for this layer is above room temperature. The FM behavior is further confirmed when measuring the isotherm magnetization, where a clear hysteretic behavior is observed, see Fig. 3.

Figure 3 (left and right) shows the magnetization versus field reversal $(M-H)$ measured at 5 and $300 \mathrm{~K}$, respectively, for all the samples. Hysteretic behavior is only observed in

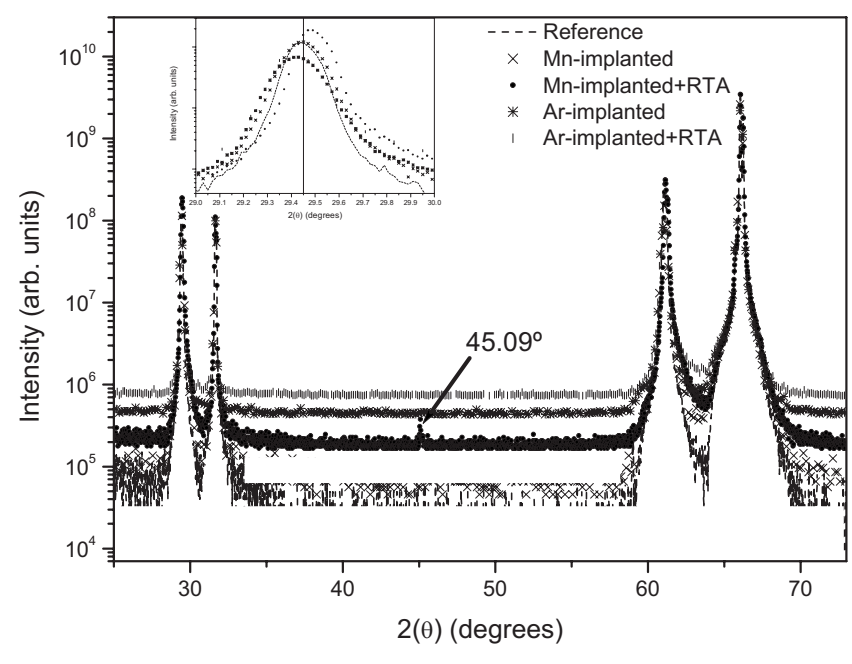

FIG. 4. Logarithmic scale picture of the XRD patterns for the reference (black dashed line), Mn-implanted (crosses), Mn-RTA (black dots), Arimplanted (stairs), and Ar-RTA (transversal black lines) samples. The insets show a zoom of the (200) InAs peak for all the previously described layers. The black line placed in the insets at $2 \theta=29.455^{\circ}$ corresponds to the diffraction angle for an ideal bulk InAs sample.

the Mn-RTA sample at both temperatures. The saturation moment $\left(M_{s}\right)$ and the coercive field $\left(H_{c}\right)$ for this sample are 7 $\times 10^{-4} \mathrm{emu} / \mathrm{m}^{-3}$ and $2 \times 10^{-2}$ Oe at $5 \mathrm{~K}$ and 5.1 $\times 10^{-4} \mathrm{emu} / \mathrm{m}^{-3}$ and $1.5 \times 10^{-2}$ Oe at $300 \mathrm{~K}$, respectively.

A characterization of the structural properties of the samples has been done in order to investigate the changes after implantation and annealing as well as the possible appearance of secondary phases and see the relation between structure and magnetism in these samples, if any. XRD patterns for as-grown, Mn- Ar-implanted, and postannealed layers are shown in Fig. 4. The four Bragg peaks observed in all XRD patterns indicate that films are composed of an InAs layer oriented along the (100) direction grown on a (100) GaAs substrate. An additional small peak located at $2 \theta$ $=45.09^{\circ}$ is observed for the Mn-RTA layer, which indicates the appearance of a secondary phase. This secondary phase is neither a Mn-As, Mn-As-In, nor In-Mn combination, since according to the JCPDS database, none of these phases present diffraction maxima around $d=2.01 \AA$.

The inset in Fig. 4 shows small changes in width and $2 \theta$ position of the (200) InAs peak for implanted and postan-
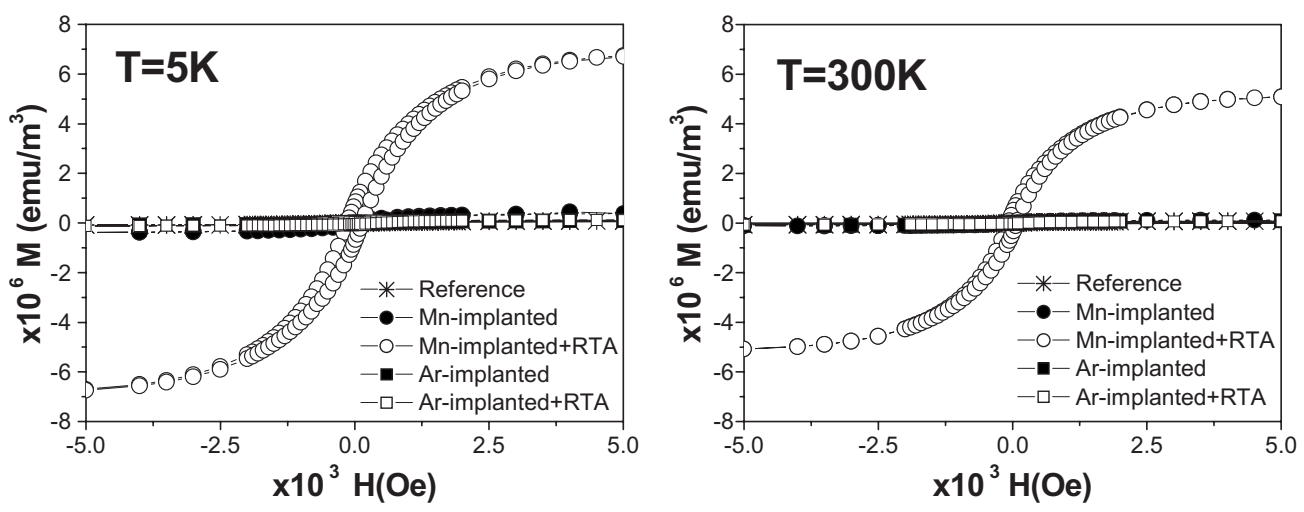

FIG. 3. Magnetization vs field reversal $(M-H)$ measured at $5 \mathrm{~K}$ (right) and $300 \mathrm{~K}$ (left) for reference (black stairs), Mn-implanted (black circles), Mn-RTA (open circles), Ar-implanted (black squares), and Ar-RTA (open squares) samples. 
nealed layers in comparison to the reference film. These changes are related to implantation-induced damage and damage recovery after RTA. As shown in the inset in Fig. 4 the (200) InAs peak for the Mn- and Ar-implanted layer became broader than in the as-grown sample, indicating a higher lattice disorder in implanted layers. After annealing the peak width slightly decreases in comparison to that of implanted layers, but it is still broader than the reference one, meaning that annealing under these conditions recovers only partially the implantation-induced damage. No shift in the (200) InAs peak position is detected for the Mn-implanted layer, which indicates that $\mathrm{Mn}$ implantation does not induce any additional stress in the InAs layer. This suggest, as next corroborated by PIXE measurement, that Mn occupies substitutional positions into the InAs lattice, whereas the peak shifts to higher angles for the Mn-RTA. A completely different situation is observed for the Ar-implanted and Ar-RTA layers, where a shift to lower angles in the (200) InAs peak position is observed.

In order to determine the location of $\mathrm{Mn}$ atoms in the InAs lattice in the Mn-implanted and Mn-RTA samples, RBS and PIXE measurements were performed in channeling configuration along the $\langle 100\rangle$ and $\langle 110\rangle$ axes. These measurements also allow us to check for the damage induced in the InAs lattice by implantation and damage recovery after annealing. ${ }^{18}$ Because of the similarity in mass numbers of $\mathrm{Mn}$ and $\mathrm{Ga}$, RBS data do not allow to resolve the Mn and Ga signals, which can be done well by PIXE. Therefore, PIXE was used for the determination of the Mn location in the film.

PIXE angular scan curves along $\langle 100\rangle$ and $\langle 110\rangle$ crystal axes taken from the In $L$, Mn $K \alpha+K \beta$, and As $K \beta$ for Mnimplanted and postannealed layers are shown in Fig. 5. For comparison an angular scan curve corresponding to a reference as-grown sample is also shown. The degree of lattice order of the as-grown layer is inferred from the measured minimum yield $\left(\chi_{\min }\right)$. In the as-grown layer the minimum yield for In at the surface is significantly higher than that expected for a typical single crystal, $\chi_{\min }=0.02-0.03,{ }^{18}$ which indicates that the surface of the $250 \mathrm{~nm}$ InAs layer is still affected by the typical defects such as misfit dislocations at the heterojunction, being not fully free of stress or defects. ${ }^{19,20}$

The large differences observed in $\chi_{\min }$ from In and from As atoms along the $\langle 100\rangle$ and $\langle 110\rangle$ axes are due to the fact that the As signal obtained by PIXE comes from the As atoms of the InAs layer and also from the GaAs substrate. Indeed, RBS measurements carried out in channeling configuration (not shown) which offers depth resolution, reveal only small differences in $\chi_{\min }$ for the In and As atoms located in the InAs layer. For this reason and in order to obtain information only about the InAs layer, we will consider for the analysis of PIXE data only the In and Mn signals.

Following room-temperature implantation the scan curves for Mn and In atoms along the $\langle 100\rangle$ and $\langle 110\rangle$ axes almost overlap, indicating that most of the Mn-implanted atoms occupy subsbtitutional positions into the In site. The quantification of the percentage of In atoms displaced from their lattice position after implantation is estimated from the
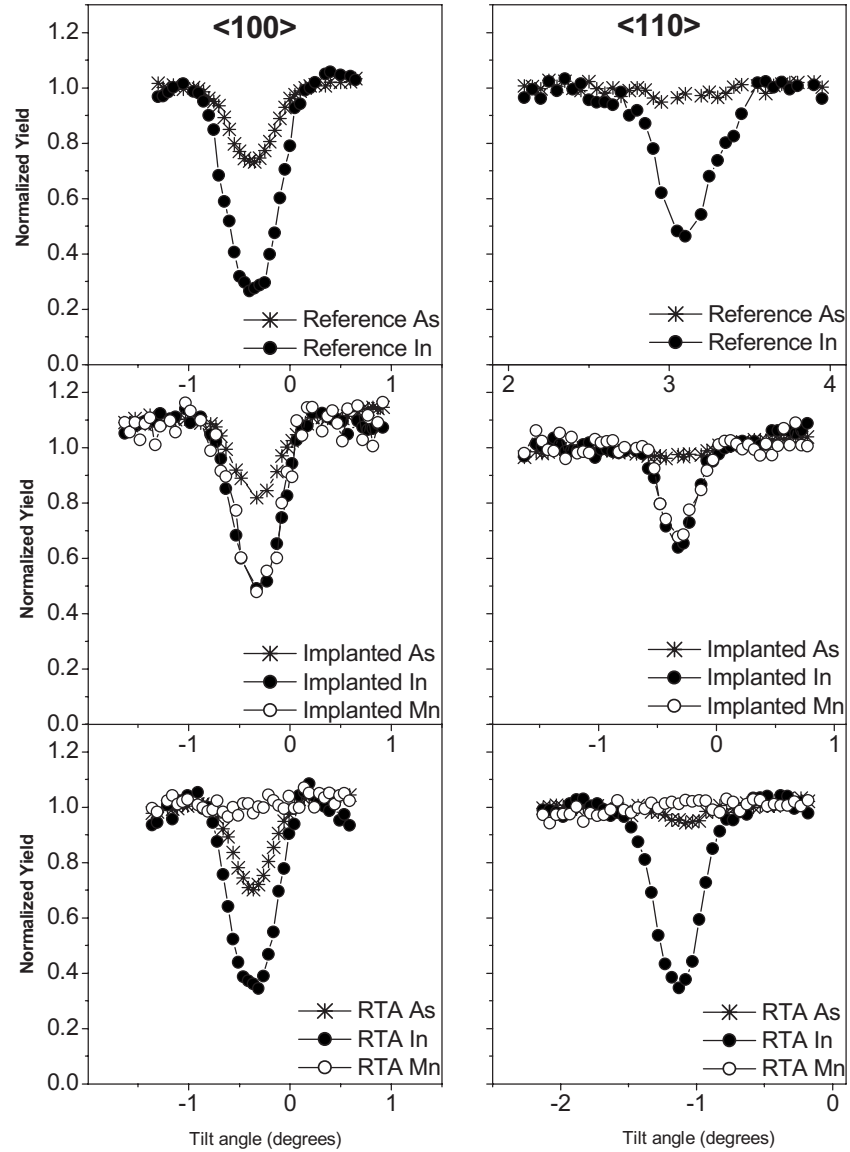

FIG. 5. PIXE angular scan curves along the $\langle 100\rangle$ (right) and $\langle 110\rangle$ (left) axes of the As $K \beta$ (crosses), In $L$ (black circles), and Mn $K \alpha+K \beta$ (open circles) lines for the reference, as-grown, (top graphs), Mn-implanted (middle graphs), and Mn-RTA (bottom graphs) layers.

$\chi_{\min }$ value. As observed in Fig. $5 \chi_{\min }$ for In atoms increases up to 0.52 for $\langle 100\rangle$ and 0.64 for $\langle 110\rangle$, in comparison to 0.26 for $\langle 100\rangle$ and 0.48 for $\langle 110\rangle$ measured for the as-grown sample, which indicates that approximately $26 \%$ for the $\langle 100\rangle$ and $18 \%$ for the $\langle 110\rangle$ of the In atoms were displaced from their lattice position, being randomly distributed in the InAs lattice after implantation.

According to the angular scan curves, annealing drives the $\mathrm{Mn}$ atoms to move away from lattice positions to be randomly distributed in the InAs lattice. Moreover, the thermal treatment promotes damage improvement, reducing $\chi_{\text {min }}$ observed for In after implantation. As deduced from the changes in $\chi_{\min }(0.36$ for $\langle 100\rangle$ and 0.35 for $\langle 110\rangle)$ after annealing, damage recovery proves to be an anisotropic process. The fact that along the $\langle 110\rangle$ axis $\chi_{\min }$ for the annealed layer is smaller than that for the as-grown one would indicate that not only the radiation damage is diminished but also part of lattice disorder induced by the as-grown defects, i.e., dislocations, whereas along the $\langle 100\rangle$ axis only a small fraction of the radiation-induced damage $(16 \%)$ is reduced. Thus, after annealing around $36 \%$ of the In atoms are still randomly distributed.

RBS data indicate that even sequential Mn implantation at fluences as high as $8 \times 10^{16} \mathrm{~cm}^{-2}$ produced high sputtering of the InAs layer, reducing up to a factor of 0.6 its thick- 
ness. Therefore, the density of Mn atoms that can be implanted into the InAs layer is limited by its low radiation resistance.

In order to eliminate the possibility that the magnetism observed at room temperature is due to lattice disorder instead of the segregation of secondary phases of Mn, a set of samples was implanted with Ar, which is inert, at the same fluences and under conditions that according to SRIM calculations ${ }^{17}$ give rise to similar damage patterns as for $\mathrm{Mn}$ (similar densities of atomic displacement and vacancy creation). As shown in Figs. 2 and 3 neither the Ar-implanted nor the Ar-RTA layer samples present any magnetic behavior, indicating that magnetism is not related to lattice disorder but to magnetic dopants and, in particular, to the formation of a Mn-based second phase.

The depth distribution of the randomly distributed $\mathrm{Mn}$ atoms in the Mn-RTA layer can be inferred by comparing experimental and simulated RBS spectra. These data show that after the thermal treatment, manganese is not uniformly distributed along the whole InAs layer, but it is segregated near to or at the film surface. Thus, Mn is supposed to be in direct contact with air and therefore, the combination of $\mathrm{Mn}$ with $\mathrm{O}$ should also be taken into account. Indeed, the elemental composition of this $\mathrm{Mn}-\mathrm{O}$ surface layers is estimated from RBS data to be 34 at. $\% \mathrm{Mn}$ and 66 at. \% O which corresponds to a general formula of $\mathrm{MnO}_{1.94}$. Bearing in mind that a $\mathrm{Mn}-\mathrm{O}$ phase could be formed, we check the $\mathrm{x}$-ray database and one $\mathrm{Mn}-\mathrm{O}$ phase presents a diffraction maximum at $2 \theta=45.09^{\circ}$, the oxygen-deficient $\mathrm{MnO}_{1.937}$ phase ${ }^{21}$ the oxygen concentration of which is close to the obtained value by RBS. The segregated $\mathrm{MnO}_{1.94}$ nanoparticles must be oriented along the (100) direction, the same as the GaAs substrate and the InAs film. This can be deduced by the fact that the (400) X-ray diffraction maximum is the only one observed and this maximum does not correspond to the more intense peak for $\mathrm{MnO}_{2-x}$ phase in polycrystalline form. The only way (004) maximum is the more intense is that the particles are oriented along the (100) direction and not randomly. This phase is the cubic defect spinel $\lambda-\mathrm{MnO}_{2}$. This oxygen-deficient $\mathrm{MnO}_{2}$ polymorph has a lattice constant of $8.04 \AA$ and a space group of $F d 3 m$. Given that the only sample which exhibits a FM behavior is the one containing this peak, the magnetic response might be due to this phase. Since the phase is oxygen deficient, $\mathrm{Mn}^{3+}$ and $\mathrm{Mn}^{4+}$ should coexist in the sample. The coexistence of $\mathrm{Mn}^{3+}-\mathrm{O}-\mathrm{Mn}^{4+}$ bonds is known to be responsible for FM via the double-exchange mechanism in different compounds, ${ }^{3,22,23}$ which usually exhibits a large Curie temperature $\left(T_{C}\right)$ even above room temperature. ${ }^{24}$ This behavior is similar to what has been previously observed for the $\mathrm{ZnO} / \mathrm{MnO}_{2}$ system. $^{3}$ Another possible explanation of the magnetism observed can be consistent with the geometric frustration inherent in the Mn sublattice in this phase. ${ }^{25,26}$ These experimental data contrast somewhat with those described in literature in which the FM behavior at room temperature of $\operatorname{In}_{1-x} \mathrm{Mn}_{x}$ As layers has been traditionally associated with the formation of magnetic MnAs clusters ${ }^{10}$ or to the doping introduction of Mn inside the InAs structure.

\section{CONCLUSIONS}

The magnetic properties of Mn- and Ar-implanted and postannealed (Mn-RTA and Ar-RTA) InAs layers grown by MBE have been investigated. Mn-InAs samples where $\mathrm{Mn}$ is substitutional in the In site behave diamagnetically. Roomtemperature FM-like behavior was only present in the MnRTA layer. The magnetic behavior of this layer (in which the $\mathrm{Mn}$ atoms are not substitutional inside the InAs lattice and mainly located near to or at the InAs surface layer) could be related to the formation of a second phase which has been identified from RBS and XRD to be an oxygen-deficient $\lambda-\mathrm{MnO}_{1.94}$ phase. Changes in the width and position of Bragg diffraction peaks and in the $\chi_{\min }$ value in the PIXE angular scan curves evidence implantation-induced lattice disorder in the InAs lattice, which is partially or fully recovered after annealing. No significant stress is developed in Mn-implanted layers in which almost all Mn atoms occupy substitutional positions at the In site, while a tensile stress is observed for the Mn-RTA layer in which the Mn atoms are segregated. A compressive stress has been measured in the Ar-implanted samples as well as in the Ar-RTA layer. The density of the Mn atoms which can be introduced via implantation in the InAs layer is limited by its low radiation resistance. By comparing magnetic and structural data for $\mathrm{Mn}$ - and Ar-implanted layers, no relation between magnetism and lattice disorder has been detected.

\section{ACKNOWLEDGMENTS}

This work was supported by CSIC Grant Nos. 200650F0122 and CSIC 2007-50I015 and in part by Grant No. MAT2005-06024-C02-01. R.G.A. acknowledges the MEC and CSIC for the Juan de la Cierva financial support. The PIXE measurements have been supported by the EU"Research Infrastructures Transnational Access" program AIM "Center for Application of Ion Beams in Materials Research" under EC Contract No. 025646.

${ }^{1}$ G. A. Prinz, Science 282, 1660 (1998).

${ }^{2}$ T. Dietl, H. Ohno, F. Matsukura, J. Cibert, and D. Ferrand, Science 287, 1019 (2000).

${ }^{3}$ M. A. García, M. L. Ruiz-González, A. Quesada, J. L. Costa-Krämer, J. F. Fernández, S. J. Khatib, A. Wennberg, A. C. Caballero, M. S. MartínGonzález, M. Villegas, F. Briones, J. M. González-Calbet, and A. Hernando, Phys. Rev. Lett. 94, 217206 (2005).

${ }^{4}$ A. Quesada, M. A. Garcia, M. Andres, A. Hernando, J. F. Fernandez, A. C. Caballero, M. S. Martin-Gonzalez, and F. Briones, J. Appl. Phys. 100, 113909 (2006).

${ }^{5}$ J. M. D. Coey, Curr. Opin. Solid State Mater. Sci. 10, 83 (2006).

${ }^{6}$ M. S. Martín-González, J. F. Fernández, F. Rubio-Marcos, I. Lorite, J. L. Costa-Krämer, A. Quesada, M. A. Bañares, and J. L. G. Fierro, J. Appl. Phys. 103, 083905 (2008).

${ }^{7}$ A. J. Blattner and B. W. Wessels, Appl. Surf. Sci. 221, 155 (2004).

${ }^{8}$ P. T. Chiu, B. W. Wessels, D. J. Keavney, and J. W. Freeland, Appl. Phys. Lett. 86, 072505 (2005)

${ }^{9}$ H. Ohno, Science 281, 951 (1998).

${ }^{10}$ H. Munekata, H. Ohno, S. von Molnar, L. L. Armin, S. Chang, and L. Esaki, Phys. Rev. Lett. 63, 1849 (1989).

${ }^{11}$ P. J. Wellmann, W. V. Schoenfeld, J. M. Garcia, and P. M. Petroff, J. Electron. Mater. 27, 1030 (1998).

${ }^{12}$ J. Shi, J. M. Kikkwa, D. D. Awschalom, G. Medeiros-Ribeiro, P. M. Petroff, and K. Babcock, J. Appl. Phys. 79, 5296 (1996).

${ }^{13}$ N. Theodoropulo, F. Hebard, S. N. G. Chu, M. E. Overberg, C. R. Abernathy, S. J. Pearton, R. G. Wilson, and J. M. Zavada, J. Appl. Phys. 91, 7499 (2002) 
${ }^{14}$ H. Ofuchi, T. Kubo, M. Tabuchi, Y. Takeda, F. Matsukura, S. P. Guo, A. Shen, and H. Ohno, J. Appl. Phys. 89, 66 (2001).

${ }^{15}$ A. Krol, Y. L. Soo, S. Huang, Z. H. Ming, Y. H. Kao, H. Munekata, and L. L. Chang, Phys. Rev. B 47, 7187 (1993).

${ }^{16}$ F. Briones, L. González, and A. Ruiz, Appl. Phys. A: Solids Surf. 49, 729 (1989).

${ }^{17}$ J. F. Ziegler, M. D. Ziegler, and J. P. Biersack, SRIM-2006.02.

${ }^{18}$ J. R. Tesmer and M. Nastasi, Handbook of Modern Ion Beam Materials Analysis (MRS, Pittsburgh, 1995).

${ }^{19}$ C. Chang, C. M. Serrano, L. C. Chang, and L. Esaki, Appl. Phys. Lett. 37, 538 (1980)

${ }^{20}$ R. S. Williams, B. M. Paine, W. J. Schaffer, and S. P. Kowalczyk, J. Vac.
Sci. Technol. 21, 386 (1982).

${ }^{21}$ JCPDS File No. 421169.

${ }^{22}$ M. B. Salamon and M. Jaime, Rev. Mod. Phys. 73, 583 (2001).

${ }^{23}$ J. Alonso, A. Arroyo, J. M. González-Calbet, M. Vallet-Regi, J. L. Martínez, J. M. Rojo, and A. Hernando, Phys. Rev. B 64, 172410 (2001).

${ }^{24}$ T. Okuda, A. Asamitsu, Y. Tomioka, T. Kimura, Y. Taguchi, and Y. Tokura, Phys. Rev. Lett. 81, 3203 (1998).

${ }^{25}$ J. E. Greedan, N. P. Raju, A. S. Wills, C. Morin, and S. M. Shaw, Chem. Mater. 10, 3058 (1998).

${ }^{26}$ N. Wang, X. Cao, G. Lin, and Y. Shihe, Nanotechnology 18, 475605 (2007). 